\title{
Prevalence of high-burden medical conditions and health care resource utilization and costs among adults with cerebral palsy
}

This article was published in the following Dove Press journal:

Clinical Epidemiology

\author{
Daniel G Whitney ${ }^{1,2}$ \\ Neil S Kamdar ${ }^{2-5}$ \\ Sophia $\mathrm{Ng}^{2}$ \\ Edward A Hurvitz' \\ Mark D Peterson ${ }^{1,2}$
}

'Department of Physical Medicine and Rehabilitation, University of Michigan, Ann Arbor, MI, USA; ${ }^{2}$ Institute for Healthcare Policy and Innovation, University of Michigan, Ann Arbor, MI, USA; ${ }^{3}$ Department of Obstetrics and Gynecology, University of Michigan, Ann Arbor, MI, USA; ${ }^{4}$ Department of Surgery, University of Michigan, Ann Arbor, MI, USA; ${ }^{5}$ Department of Emergency

Medicine, University of Michigan, Ann Arbor, MI, USA
Correspondence: Daniel G Whitney Department of Physical Medicine and Rehabilitation, University of Michigan, 325 E. Eisenhower Pkwy, Ann Arbor, MI 48I08, USA

$\mathrm{Tel}+I 7349367175$

Email dgwhit@umich.edu
Purpose: Individuals with cerebral palsy (CP) are susceptible to early development of highburden medical conditions, which may place a considerable strain on health care resources. However, little is known about the prevalence of high-burden medical conditions or health care resource utilization among adults with CP. The purpose of this study was to determine the prevalence of high-burden medical conditions and health care resource utilization and costs among adults with $\mathrm{CP}$, as compared to adults without $\mathrm{CP}$.

Patients and methods: Cross-sectional data from the 2016 Optum Clinformatics ${ }^{\circledR}$ Data Mart, a de-identified nationwide claims database of beneficiaries from a single private payer in the US. ICD-10-CM diagnosis codes were used to identify all medical conditions among beneficiaries with and without CP who were between 18 and 64 years of age. Medical and outpatient pharmacy claims were used to identify annual all-cause health care resource utilization and health care costs as standardized reimbursement and patient out-of-pocket costs.

Results: Adults with CP $(\mathrm{n}=5,555)$ had higher prevalence and odds of all medical conditions compared to adults without $\mathrm{CP}(\mathrm{OR}=1.3-5.8$; all $P<0.05)$, except cancer $(\mathrm{OR}=1.1 ; 95 \%$ $\mathrm{CI}=0.9-1.3$ ). Adults with $\mathrm{CP}$ had greater annual mean counts of all health care service types (eg, inpatient, emergency department) compared to adults without $\mathrm{CP}$ (all $P<0.01$ ). Adults with CP had higher unadjusted standardized reimbursement (mean difference $=\$ 16,288$; cost ratio $[\mathrm{CR}]=3.0 ; 95 \% \mathrm{CI}=2.9-3.1$ ) and patient out-of-pocket (mean difference $=\$ 778 ; \mathrm{CR}=1.7$; 95\% CI=1.6-1.7) costs compared to adults without CP. After adjusting for all prevalent medical conditions, adults with $\mathrm{CP}$ still had higher standardized reimbursement $(\mathrm{CR}=2.5$; 95\% $\mathrm{CI}=2.5-2.6)$ and patient out-of-pocket $(\mathrm{CR}=1.8 ; 95 \% \mathrm{CI}=1.7-1.8)$ costs.

Conclusion: Adults with $\mathrm{CP}$ have a higher prevalence of high-burden medical conditions, health care resource utilization, and health care costs compared to adults without CP. Study findings suggest the need for earlier screening strategies and preventive medical services to quell the disease and economic burden attributable to adults with $\mathrm{CP}$.

Keywords: cerebral palsy, epidemiology, disease, burden

\section{Introduction}

Cerebral palsy (CP) is a neurological syndrome that represents a heterogeneous group of movement disorders. CP is the most common pediatric physical disability, with prevalence estimates of 3.1 per 1,000 children. ${ }^{1}$ Causes of CP can vary from genetic predisposition, brain lesions, and environmental exposures that negatively affect brain development. The heterogeneous and medically complex nature of CP is due to many factors, such as timing and cause of $\mathrm{CP}$, as well as comorbidities (eg, epilepsy, intellectual disabilities); however, the common feature of $\mathrm{CP}$ is the potential for 
restrictions in activities of daily living and societal integration, as well as the increased risk for health complications.

Children with $\mathrm{CP}$ are more likely to experience an array of health and function problems compared to children without CP, including orthopedic-related issues, ${ }^{2}$ excess body fat deposition, ${ }^{2-4}$ and mental health disorders. ${ }^{5}$ Although CP is considered a "non-progressive" neurological condition, CP is a chronic and lifelong condition with many people reaching normal life expectancies. ${ }^{6}$ Having complex health care needs and health complications throughout growth could have lasting ramifications on the health status of the adult. Research has shown that health and function problems may increase in severity as children with CP transition into and throughout their adult years. ${ }^{7-10}$ Consequently, adults with $\mathrm{CP}$ are at risk of early development of several medical conditions that incur excess health care costs and represent a considerable patient and caregiver burden, including chronic pain, ${ }^{11}$ heightened fracture susceptibility, ${ }^{8}$ and non-communicable diseases (eg, cardiometabolic, mental health). ${ }^{9,10,12,13}$

There is very little knowledge about the life-course health development and healthy aging process among adults with $\mathrm{CP}$, resulting in insufficient clinical care for these populations. At present, the US health care system is not well-designed to meet the greater demands imposed by individuals with neurological conditions, ${ }^{14,15}$ thus further increasing risk of health disparities that may otherwise be prevented or lessened. Importantly, the population of adults with $\mathrm{CP}$ is projected to expand in the coming decades, which may lead to a disproportionate increase in the burden of disease and economic burden attributable to CP. Therefore, characterizing the prevalence of highburden medical conditions and a comprehensive understanding of the health care economic burden among adults with CP is crucial, especially among young and middleaged adults. Such information could serve to assist health care policy reform and also to inform better clinical practice guidelines that facilitate earlier, long-term, and costeffective care for the aging population with CP. Using a single private payer administrative claims database, the objective of this study was to determine the prevalence of several high-burden medical conditions and to descriptively characterize health care resource utilization and costs among young and middle-aged adults with $\mathrm{CP}$, as compared to young and middle-aged adults without CP. We hypothesized that adults with $\mathrm{CP}$ would have a higher prevalence of all medical conditions, higher health care resource utilization, and higher health care costs compared to adults without $\mathrm{CP}$.

\section{Material and methods Data source}

Data were collected from the Clinformatics ${ }^{\circledR}$ Data Mart Database (OptumInsight ${ }^{\mathrm{TM}}$, Eden Prairie, MN, USA). This is a nationwide single private payer administrative claims database with de-identified data of 79 million beneficiaries who have commercial or Medicare Advantage health plans from 2001-2017. These private payer administrative claims data include service utilization throughout enrollment on their insurance plan. Data are de-identified and the University of Michigan Institutional Review Board approved this study as non-regulated. The investigators (DGW, SN, NSK, and MDP) have a data use agreement to analyze this database.

\section{Sample selection}

Data were obtained from the most recent available year, 2016. Beneficiaries who were between 18 and 64 years of age, had 12 full months of continuous enrollment, and had at least one service utilization were considered for this investigation. ICD-10 codes were used to identify all medical conditions, which were identified using a single claim at any position, and are presented in Table 1. The single claim-based definition has shown good accuracy for identifying pediatriconset conditions using administrative claims data, with sensitivity of $99 \%$ and positive predictive value of $79 \% .{ }^{16}$ Data regarding severity of $\mathrm{CP}$ using common clinical measures (eg, gross motor function classification system) are not available in administrative claims. Further, more than $70 \%$ of the cohort had "other" or "unspecified" CP, thus not allowing us to stratify or account for the clinical subtypes of CP (eg, spasticity/athetoid, hemiplegic) in the current study.

\section{Medical conditions}

Prevalent high-burden medical conditions were selected with guidance from the literature on disease, disability, and mortality among adults. ${ }^{8,9,17-24}$ Medical conditions included were pain, all-cause fracture at any location, and several noncommunicable diseases grouped into the following categories: 1) ischemic heart diseases; 2) cerebrovascular diseases; 3 ) hypertensive and other cardiovascular diseases; 4) type 2 diabetes mellitus; 5) malignant cancer; 6) osteoporosis; 7) osteoarthritis; 8) mood affective disorders; 9) anxiety disorders; 10) chronic kidney diseases; and 11) liver diseases.

\section{Health care resource utilization}

Annual all-cause health care resource utilization was identified using medical claims and categorized into the following 
Table I ICD-10 codes used to identify all diagnoses

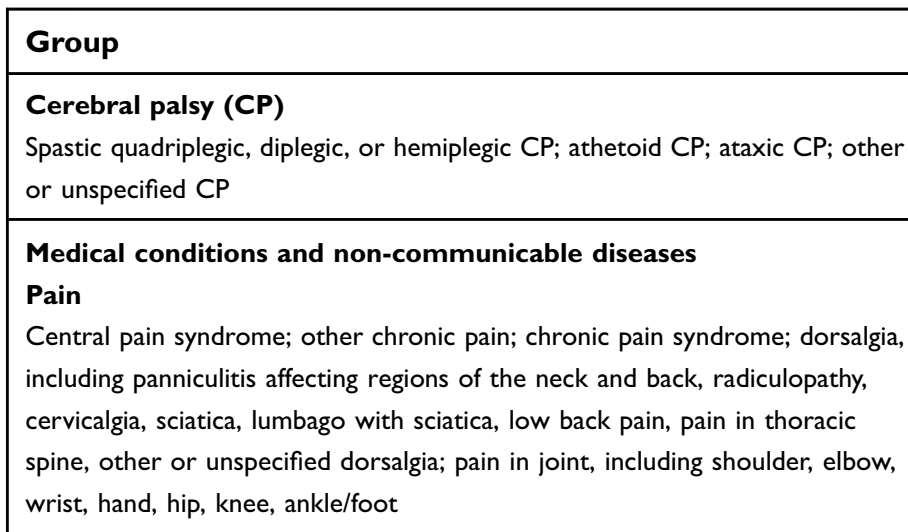

\section{Fracture}

Osteoporosis with current pathological fracture; fracture of skull and facial bones, cervical vertebra and other parts of neck, rib(s), sternum, and thoracic spine, lumbar spine and pelvis, shoulder and upper arm, forearm, wrist and hand, femur, lower leg, including ankle, foot and toe, except ankle

\section{Ischemic heart disease}

Angina pectoris; acute myocardial infarction; subsequent ST elevation (STEMI) and non-ST elevation (NSTEMI) myocardial infarction; other acute ischemic heart diseases; chronic ischemic heart disease

\section{Cerebrovascular disease}

Nontraumatic subarachnoid or intracerebral hemorrhage; other and unspecified nontraumatic intracranial hemorrhage; cerebral infarction; occlusion and stenosis of precerebral or cerebral arteries, not resulting in cerebral infarction; other cerebrovascular diseases; cerebrovascular disorders in diseases classified elsewhere; sequelae of cerebrovascular disease

\section{Hypertensive and other cardiovascular disease}

Essential (primary) hypertension; hypertensive heart, chronic kidney disease, or heart and chronic kidney disease; secondary hypertension; hypertensive crisis; heart failure; peripheral atherosclerosis

\section{Type 2 diabetes mellitus}

\section{Malignant cancer}

Malignant neoplasms of lip, oral cavity, or pharynx, digestive organs, respiratory and intrathoracic organs, bone and articular cartilage, mesothelial and soft tissue, breast, female or male genital organs, urinary tract, eye, brain, or other parts of central nervous system, thyroid or other endocrine glands, illdefined, other secondary, or unspecified sites, neuroendocrine tumors, lymphoid, hematopoietic, or related tissue; melanoma and other malignant neoplasms of skin

\section{Osteoporosis}

With or without current pathological fracture

\section{ICD-10 codes}

G80 family

G890, G8929, G894, M54 family, M255 family

M80 family, S02 family, SI 2 family, S22 family, S32 family, S42

family, S52 family, S62 family, S72 family, S82 family, S92 family

120-22 families, 124 family, 125 family

160-63 families, 165-69 families

II0-I3 families, II5 family, II6 family, I50 family, I70 family

EII family

C00-26 families, C30-4I families, C43-58 families, C60-80

families, C7A family, C7B family, C8I-96 families

\section{Osteoarthritis (OA)}

Poly OA; hip OA; knee OA; OA of the first carpometacarpal joint; other and unspecified OA

\section{Mood affective disorders}


Table I (Continued).

\begin{tabular}{|l|l|}
\hline Group & ICD-IO codes \\
\hline $\begin{array}{l}\text { Manic episode; bipolar disorder; major depressive disorder, single episode or } \\
\text { recurrent; persistent mood (affective) disorders; unspecified mood (affective) } \\
\text { disorders }\end{array}$ & F30-34 families, F39 \\
\hline $\begin{array}{l}\text { Anxiety disorders } \\
\text { Phobic anxiety disorders; other anxiety disorders; obsessive-compulsive dis- } \\
\text { order; reaction to severe stress, and adjust disorders; dissociative and con- } \\
\text { version disorders; somatoform disorders; other nonpsychotic mental } \\
\text { disorders }\end{array}$ & F40-45 families, F48 family \\
\hline $\begin{array}{l}\text { Chronic kidney disease } \\
\text { Stage I-V; end stage renal disease; chronic kidney disease, unspecified }\end{array}$ & NI8 family \\
\hline $\begin{array}{l}\text { Liver disease } \\
\text { Alcoholic liver disease; toxic liver disease; hepatic failure, not elsewhere } \\
\text { classified; chronic hepatitis, not elsewhere classified; fibrosis and cirrhosis of } \\
\text { liver; other inflammatory liver diseases; other diseases of liver; liver disorders } \\
\text { in diseases classified elsewhere }\end{array}$ & K70-76 families, K77 \\
\hline
\end{tabular}

service types: inpatient; outpatient, ambulatory, and other (herein referred to as "outpatient and other"); emergency department; office; and ancillary. Specific services per service category are presented in Table 2. Measures to assess health care resource utilization included the percentage of patients that had at least one service utilization and the mean count of service utilization. To limit extreme values and to reduce the effect of possible spurious outliers, winsorization of data to the upper $99.9 \%$ was performed for each service category.

\section{Health care costs}

Annual all-cause health care costs were identified using medical and outpatient pharmacy claims, and were from the paid amounts of adjudicated claims. The adjudicated claims included payer payments and patient copayments, deductibles, and coinsurance. Medical, outpatient pharmacy, and total (medical + outpatient pharmacy) all-cause health care costs were represented as standardized reimbursement (sum of all health plan and patient paid amounts) and patient out-ofpocket (sum of copayment, deductible, and coinsurance) costs.

To account for differences in pricing across health plans and provider contracts, the standardized cost field involves algorithms that reflect the allowed payment amounts across all provider services. This allows comparisons across patients, data sources, and geographic areas, and accounts for contractual payer and provider differences. Price standardization accounts for quantity of services provided, relative resource costs involved in providing the services, and the nature of the service which can be defined as the CPT/ HCPCS codes for professional services, NDC for pharmacy service, or type of admission for the inpatient stay.

\section{Sociodemographic variables}

Age, sex, ethnicity, education level, household annual income, and insurance coverage (ie, commercial only, Medicare Advantage) were available from the database and were considered for risk adjustment.

\section{Statistical analysis}

Descriptive characteristics, medical conditions, and health care resource utilization and cost measures were summarized using mean (SD) for continuous variables and percentage $(95 \% \mathrm{CI})$ for categorical variables.

Chi-squared tests were performed to determine group differences for each of the medical conditions. Then, we constructed multivariable logistic regression models to determine the OR for each medical condition with group (reference: without $\mathrm{CP}$ ) as the primary exposure variable, while controlling for age and sex. The main effect of group was interpreted. Since OR does not approximate relative risk when outcomes have prevalence estimates of more than $10 \%$ and an adjusted OR more than 2.5 , we applied a correction formula for outcomes that met both criteria to more appropriately interpret the relative risk, as previously described. $^{25}$ 
Table 2 Health care resource utilization by service type

\begin{tabular}{|l|l|}
\hline Health care service type & Level I Description: Level 2 Description \\
\hline Inpatient services & Facility Inpatient: Acute and Rehab/Skilled Nursing Facility; Professional Services: Inpatient Visits \\
\hline $\begin{array}{l}\text { Outpatient, ambulatory, and } \\
\text { other services }\end{array}$ & $\begin{array}{l}\text { Facility Outpatient (OP): OP Facility Diagnostic, Laboratory, Other, Radiology, and Surgery; Professional } \\
\text { Services: Allergy Tests and Injections, Anesthesia, Consultations, Diagnostic Testing, Immunizations and } \\
\text { Injections, Laboratory, Mental Health, Obstetrics, Pathology, Physical Medicine/Rehab, Professional Other, } \\
\text { Preventive Medicine, Radiology, Surgery, Vision, Hearing, and Speech }\end{array}$ \\
\hline Emergency department services & Facility Outpatient: Emergency Room; Professional Services: Emergency Room \\
\hline Office services & Professional Services: Office Visits \\
\hline Ancillary & $\begin{array}{l}\text { Ancillary: Durable Medical Equipment, Drugs Administered, Home Health/Hospice Visits, Services and Supplies, } \\
\text { Transportation Services }\end{array}$ \\
\hline
\end{tabular}

Unadjusted group differences for health care resource utilization measures were examined using chi-squared tests for binary data and generalized linear models assuming a zero-inflated Poisson distribution (for non-utilization) and log-link function for count data. Generalized linear models with gamma distribution and log-link function were performed to estimate the cost ratios (CRs) (exponentiated form of parameter estimate) for explanatory variables, as previously recommended. ${ }^{26-28}$ The main effect of group was interpreted before and after controlling for potential confounding factors including age, sex, insurance coverage, and all medical conditions. Adjusted models were developed for total standardized reimbursement costs and total patient out-of-pocket costs.

Logistic and generalized linear models did not control for ethnicity, education level, and household annual income to limit bias because of the extent of missingness/unknown. However, we conducted a sensitivity analysis by further adjusting the multivariable regression models for ethnicity, education level, and household annual income for individuals with complete data.

Finally, we stratified results by sex for unadjusted prevalence of medical conditions and unadjusted health care resource utilization and cost measures. All analyses were performed using SAS version 9.4 (SAS Institute Inc., Cary, NC, USA). Effect estimates were reported as ORs or CRs with $95 \%$ CI. $P \leq 0.05$ (two-tailed) was used to determine statistical significance.

\section{Results}

Descriptive characteristics and prevalence of medical conditions for adults with $\mathrm{CP}(\mathrm{n}=5,555)$ and without $\mathrm{CP}$ $(\mathrm{n}=5,531,366)$ are presented in Table 3. Adults with $\mathrm{CP}$ had a higher prevalence of all medical conditions compared to adults without CP (all $P<0.001$ ), except for malignant cancer $(P=0.74)$. The adjusted ORs for each medical condition are presented in Figure 1. After adjusting for age and sex, adults with CP had higher odds of pain, fracture, ischemic heart diseases, cerebrovascular diseases, hypertension and other cardiovascular diseases, type 2 diabetes mellitus, osteoporosis, osteoarthritis, mood affective disorders, anxiety disorders, chronic kidney diseases, and liver diseases (ORs $=1.25-5.76$; all $P<0.05)$, but not malignant cancer $(\mathrm{OR}=1.10 ; 95 \%$ $\mathrm{CI}=0.96-1.27)$.

Health care resource utilization is presented in Table 4. Adults with CP had a higher prevalence of having at least one service utilized in 2016 compared to adults without CP for inpatient visits, outpatient and other visits, emergency department visits, office visits, and ancillary use (all $P<0.001$ ). Adults with $\mathrm{CP}$ had a greater annual mean count of service utilization compared to adults without $\mathrm{CP}$ for inpatient visits ( $3.0 \pm 8.2$ vs $0.4 \pm 2.5)$, outpatient and other visits (16.6 20.9 vs $7.4 \pm 10.3)$, emergency department visits ( $1.0 \pm 1.9$ vs $0.3 \pm 0.9)$, office visits $(6.7 \pm 6.4$ vs $4.0 \pm 4.6)$, and ancillary use $(9.4 \pm 27.0$ vs $1.4 \pm 5.8)$ (all $P<0.001$ ).

Unadjusted all-cause health care costs are presented in Figure 2. The standardized reimbursement costs (Figure 2A) were higher for adults with $\mathrm{CP}$ compared to adults without $\mathrm{CP}$ for total (mean difference $=\$ 16,288 ; \quad \mathrm{CR}=2.96 ; 95 \%$ $\mathrm{CI}=2.85-3.08$ ), medical (mean difference $=\$ 13,732$; $\mathrm{CR}=3.05 ; 95 \% \mathrm{CI}=2.93-3.18$ ), and pharmacy (mean difference $=\$ 2,556 ; \mathrm{CR}=2.42 ; 95 \% \mathrm{CI}=2.30-2.53$ ). The patient outof-pocket costs (Figure 2B) were higher for adults with $\mathrm{CP}$ compared to adults without $\mathrm{CP}$ for total (mean difference= $\$ 778$; $\mathrm{CR}=1.65 ; 95 \% \mathrm{CI}=1.60-1.70$ ), medical (mean difference $=\$ 655 ; \mathrm{CR}=1.77 ; 95 \% \mathrm{CI}=1.71-1.84)$, and pharmacy (mean difference $=\$ 123 ; \mathrm{CR}=1.40 ; 95 \% \mathrm{CI}=1.35-1.45$ ). 
Table 3 Descriptive characteristics and prevalence of medical conditions among adults with and without cerebral palsy (CP)

\begin{tabular}{|c|c|c|}
\hline & $C P(n=5,555)$ & Without CP $(n=5,53 I, 366)$ \\
\hline \multicolumn{3}{|l|}{ Descriptive characteristics } \\
\hline Age, mean (SD) & $42.3(13.9)$ & $43.8(13.0)$ \\
\hline \multicolumn{3}{|l|}{ Sex, \% } \\
\hline Female & 47.9 & 53.9 \\
\hline Male & 52.2 & 46.2 \\
\hline \multicolumn{3}{|l|}{ Ethnicity, \% } \\
\hline White & 55.2 & 53.5 \\
\hline Black & 12.1 & 7.4 \\
\hline Hispanic & 8.9 & 9.4 \\
\hline Asian & 2.0 & 4.3 \\
\hline Unknown/missing & 21.8 & 25.4 \\
\hline \multicolumn{3}{|l|}{ Education, \% } \\
\hline Less than high school & 0.5 & 0.5 \\
\hline High school diploma & 32.0 & 24.0 \\
\hline More than high school & 63.3 & 73.4 \\
\hline Unknown/missing & 4.3 & 2.1 \\
\hline \multicolumn{3}{|l|}{ Household annual income, \% } \\
\hline$<\$ 40 \mathrm{~K}$ & 23.8 & 13.1 \\
\hline$\$ 40-59.9 \mathrm{~K}$ & 10.5 & 10.0 \\
\hline$\$ 60-99.9 \mathrm{~K}$ & 16.7 & 20.0 \\
\hline$\geq \$ 100 \mathrm{~K}$ & 19.6 & 34.1 \\
\hline Unknown/missing & 29.4 & 22.8 \\
\hline \multicolumn{3}{|l|}{ Insurance coverage } \\
\hline Commercial only & 41.5 & 93.1 \\
\hline Medicare Advantage & 58.5 & 6.9 \\
\hline Medical conditions ${ }^{\mathbf{a}}$ & $\%(95 \% \mathrm{Cl})$ & $\%$ \\
\hline Pain & $38.6(37.3,39.9)$ & 30.4 \\
\hline Fracture & $6.3(5.7,6.9)$ & 2.7 \\
\hline Ischemic heart disease & $5.0(4.4,5.6)$ & 3.6 \\
\hline Cerebrovascular disease & $6.8(6.1,7.5)$ & 1.6 \\
\hline Hypertensive/other cardiovascular disease & $37.4(36.1,38.7)$ & 25.6 \\
\hline Type 2 diabetes & I2.4 (II.5, I3.3) & 10.1 \\
\hline Malignant cancer & $4.0(3.5,4.5)$ & 3.9 \\
\hline Osteoporosis & $5.5(4.9,6.1)$ & 1.3 \\
\hline Osteoarthritis & $14.9(14.0,15.8)$ & 7.9 \\
\hline Mood affective disorders & $23.4(22.3,24.5)$ & 11.2 \\
\hline Anxiety disorders & $23.7(22.6,24.8)$ & 15.3 \\
\hline Chronic kidney disease & $4.3(3.8,4.8)$ & 1.9 \\
\hline Liver disease & $4.1(3.6,4.6)$ & 3.0 \\
\hline
\end{tabular}

Note: ${ }^{a}$ All medical conditions were significantly higher among adults with vs without $C P(P<0.000 \mathrm{I}$ using chi-squared test), except for malignant cancer $(P=0.74)$.

All-cause health care costs after adjusting for age, sex, insurance coverage, and all medical conditions are presented in Table 5. Adults with $\mathrm{CP}$ had higher total standardized reimbursement $(\mathrm{CR}=2.54 ; 95 \% \mathrm{CI}=2.46-2.63)$ and patient out-of-pocket $(\mathrm{CR}=1.75 ; 95 \% \mathrm{CI}=1.70-1.81)$ costs compared to adults without $\mathrm{CP}$.
The results of the sensitivity analysis $(n=3,543,139)$ found that additional covariate adjustment for ethnicity, education level, and household annual income did not alter interpretations for the main effect of group for most medical conditions that were higher in the primary analysis, and increased adjusted CRs for total 


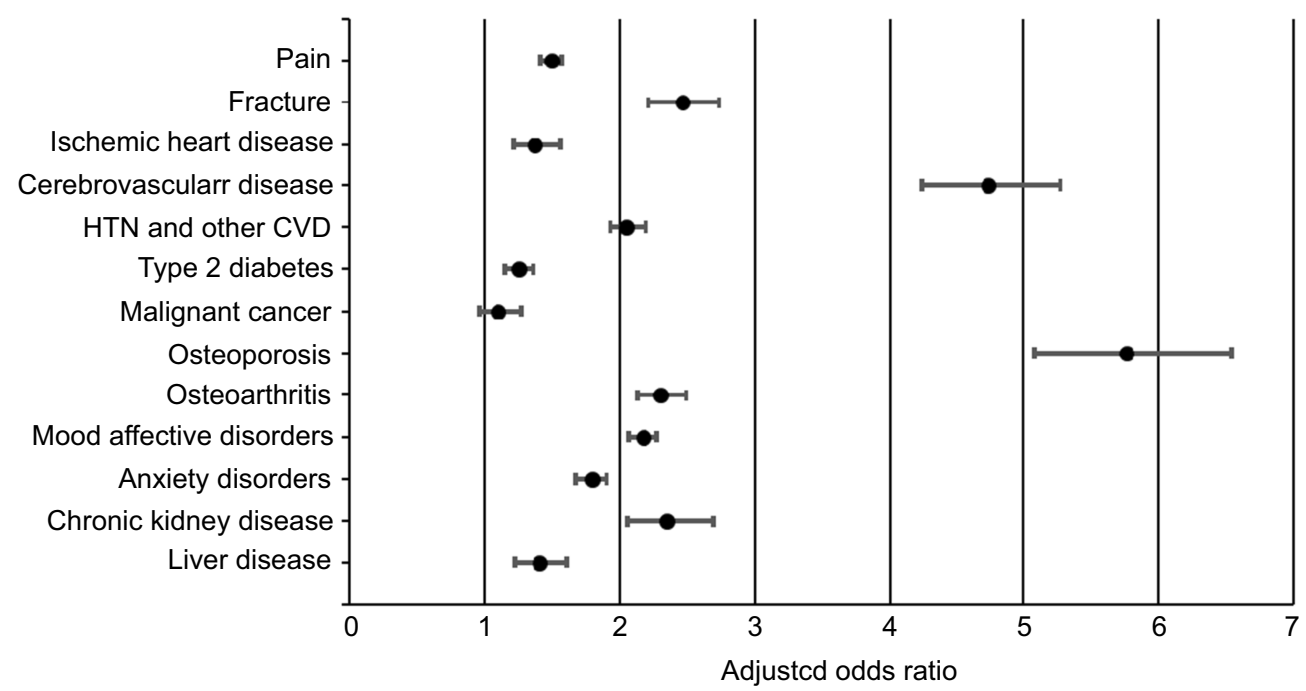

Figure I Adjusted ORs and 95\% Cls of medical conditions between adults (I8-64) with cerebral palsy (CP) compared to adults without CP (reference). ORs are adjusted for age and sex.

Abbreviations: HTN, hypertension; CVD, cardiovascular disease.

Table 4 Health care resource utilization in 2016 among adults with and without cerebral palsy (CP)

\begin{tabular}{|c|c|c|c|c|c|c|}
\hline & \multicolumn{2}{|l|}{$C P(n=5,555)$} & \multicolumn{2}{|c|}{ Without CP $(n=5,53 I, 366)$} & \multirow[b]{2}{*}{$\begin{array}{l}P \text {-value (Mean } \\
\text { count) }\end{array}$} & \multirow[b]{2}{*}{$\begin{array}{l}\text { P-value } \\
(\%)^{c}\end{array}$} \\
\hline & $\begin{array}{l}\text { Mean count } \\
\text { (SD) }\end{array}$ & $\begin{array}{l}\% \\
\text { patients }^{a}\end{array}$ & $\begin{array}{l}\text { Mean count } \\
\text { (SD) }\end{array}$ & $\begin{array}{l}\% \\
\text { patients }^{\mathrm{a}}\end{array}$ & & \\
\hline Inpatient visits & $3.0(8.2)$ & 23.7 & $0.4(2.5)$ & 7.3 & $<0.0001$ & $<0.0001$ \\
\hline $\begin{array}{l}\text { Outpatient and other } \\
\text { visits }\end{array}$ & $16.6(20.9)$ & 97.5 & $7.4(10.3)$ & 94.9 & $<0.0001$ & $<0.0001$ \\
\hline $\begin{array}{l}\text { Emergency department } \\
\text { visits }\end{array}$ & $1.0(1.9)$ & 38.4 & $0.3(0.9)$ & 19.0 & $<0.0001$ & $<0.0001$ \\
\hline Office visits & $6.7(6.4)$ & 93.1 & $4.0(4.6)$ & 85.8 & $<0.0001$ & $<0.0001$ \\
\hline Ancillary use & $9.4(27.0)$ & 74.5 & I.4 (5.8) & 35.5 & $<0.0001$ & $<0.0001$ \\
\hline
\end{tabular}

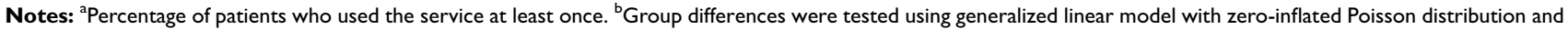
log-link function. ' $\mathrm{G}$ roup differences were tested using chi-squared tests.

standardized $\quad$ reimbursement $\quad(\mathrm{CR}=2.78 ; \quad 95 \%$ $\mathrm{CI}=2.67-2.90)$ and patient out-of-pocket $(\mathrm{CR}=1.83$; $95 \% \mathrm{CI}=1.77-1.90)$ costs. Specifically, the odds of ischemic heart diseases $(\mathrm{OR}=1.04 ; 95 \% \mathrm{CI}=0.88-1.23)$ and type 2 diabetes $(\mathrm{OR}=0.95 ; 95 \% \mathrm{CI}=0.85-1.07)$ were no longer significantly higher among adults with $\mathrm{CP}$, but the odds of all other medical conditions remained higher (ORs=1.29-5.96; all $P<0.05$ ).

Unadjusted prevalence of medical conditions and unadjusted health care resource utilization and costs stratified by sex are presented in Table 6 . The main findings were consistent across females and males with vs without $\mathrm{CP}$, except for the similar prevalence of ischemic heart diseases and type 2 diabetes among men with and without CP.

\section{Discussion}

The chief finding of this study is that privately-insured young and middle-aged adults with $\mathrm{CP}$ had higher prevalence of various high-burden medical conditions and higher economic burden compared to adults without $\mathrm{CP}$. The excess health care costs were evident even after accounting for costly medical conditions. These findings provide large, national-level data to support the need for earlier screening strategies and preventive medical services for populations with neurological conditions. ${ }^{9,13}$ Improved clinician awareness of the lifecourse health development and more efficient medical referral systems could prevent, minimize, or lessen the burden of medical conditions for patients with neurological conditions. This is particularly pertinent for populations with pediatriconset disabilities, such as $\mathrm{CP}$, as these individuals may be 

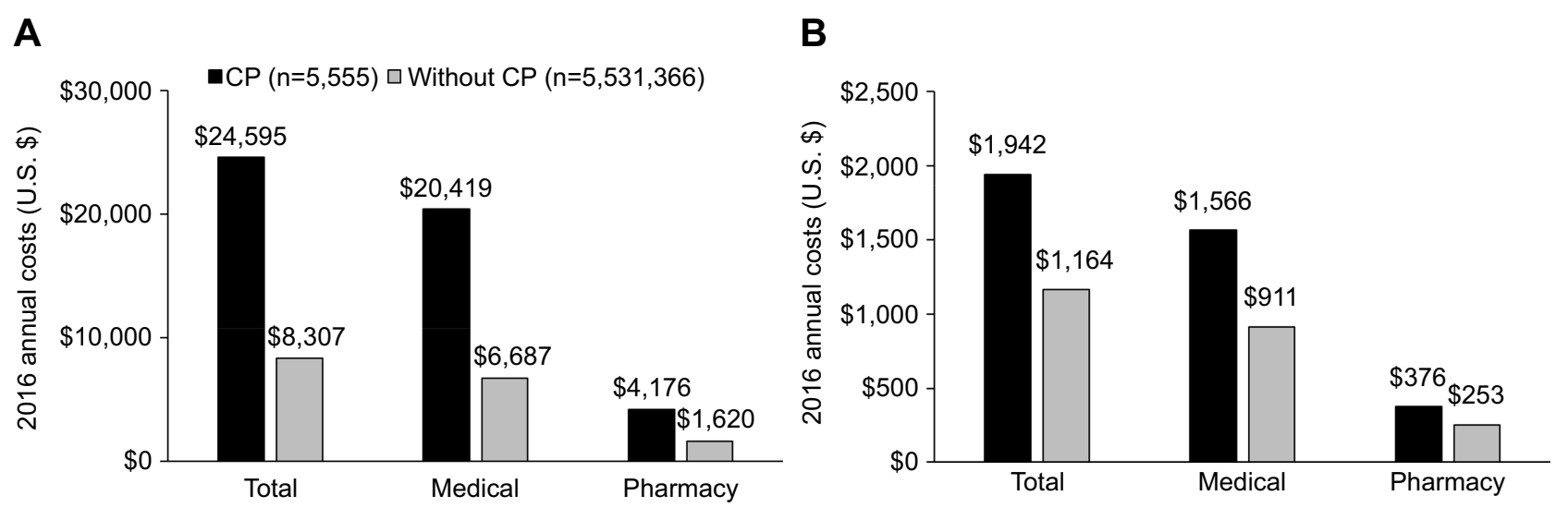

Figure 2 Mean all-cause total, medical, and pharmacy standardized reimbursement costs (A) and mean all-cause total, medical, and pharmacy patient out-of-pocket costs (B) in 2016 among adults (18-64 years) with and without cerebral palsy (CP).

Table 5 Adjusted cost ratios (CRs) in 2016 among adults with and without cerebral palsy (CP)

\begin{tabular}{|c|c|c|}
\hline & Standardized reimbursement & Patient out-of-pocket \\
\hline & CR $(95 \% \mathrm{Cl})$ & CR $(95 \% \mathrm{CI})$ \\
\hline With CP (ref: without CP) & $2.54(2.46,2.63)$ & $\mathrm{I} .75(\mathrm{I} .70, \mathrm{I} .8 \mathrm{I})$ \\
\hline Age (continuous) & $1.00(1.00,1.00)$ & $1.00(1.00,1.00)$ \\
\hline Sex (ref: female) & $0.77(0.77,0.77)$ & $0.82(0.82,0.82)$ \\
\hline Insurance coverage (ref: commercial only) & $1.38(1.37,1.38)$ & $0.72(0.7 \mathrm{I}, 0.72)$ \\
\hline Medical conditions and non-communicable & & \\
\hline Pain & $1.78(1.78,1.79)$ & $1.60(1.60,1.61)$ \\
\hline Fracture & $1.90(1.89,1.91)$ & $1.55(1.54,1.56)$ \\
\hline Ischemic heart disease & $2.05(2.04,2.06)$ & $1.55(1.54,1.56)$ \\
\hline Cerebrovascular disease & $1.98(1.96,2.00)$ & $1.42(1.41,1.43)$ \\
\hline Hypertensive/other cardiovascular disease & $1.47(1.47,1.48)$ & $1.29(1.29,1.30)$ \\
\hline Type 2 diabetes & $1.42(1.41,1.42)$ & $1.26(1.26,1.27)$ \\
\hline Malignant cancer & $3.62(3.60,3.64)$ & $1.84(1.83,1.85)$ \\
\hline Osteoporosis & $1.37(1.36,1.38)$ & $1.16(1.15,1.17)$ \\
\hline Osteoarthritis & $1.54(1.53,1.54)$ & $1.31(1.30,1.31)$ \\
\hline Mood affective disorders & $1.56(1.55,1.57)$ & $1.36(1.36,1.37)$ \\
\hline Anxiety disorders & $1.48(1.47,1.48)$ & $1.35(1.35,1.35)$ \\
\hline Chronic kidney disease & $3.23(3.21,3.26)$ & $1.49(1.48,1.50)$ \\
\hline Liver disease & $2.34(2.33,2.36)$ & $1.57(1.56,1.57)$ \\
\hline
\end{tabular}

Notes: Generalized linear models with gamma distribution and log-link function were performed to estimate adjusted CRs and $95 \%$ Cls, which is the exponentiated form of the parameter estimate. Models were adjusted for all variables in the table.

unhealthy for longer, incur higher lifetime health care costs, ${ }^{29}$ and impose greater lifetime health care resource utilization than individuals without $\mathrm{CP}$.

In the current study, while we found profound health disparities of all medical conditions, except for malignant cancer in the primary analysis, adults with CP had exceedingly higher odds of osteoporosis and cerebrovascular diseases compared to adults without CP. The findings for osteoporosis are not surprising. Children with $\mathrm{CP}$ have poor motor control ${ }^{30,31}$ and low physical activity levels, ${ }^{2}$ and are predisposed to mobility decline as they transition into and throughout adulthood. ${ }^{7}$ This inevitably leads to inadequate mechanical loading - the primary stimulus for musculoskeletal development. Indeed, children with $\mathrm{CP}$ have a blunted accretion of muscle and bone $e^{2,32}$ as early as their second year of life, ${ }^{33}$ and a higher degree of fat infiltration within skeletal muscle and bone marrow. ${ }^{2}$ This underdeveloped musculoskeletal profile not only increases poor preservation of musculoskeletal tissue and fracture risk throughout the lifespan, ${ }^{8,9}$ it is also implicated in the pathogenesis of obesity 
Table 6 Prevalence of medical conditions and health care resource utilization and costs among adults with and without cerebral palsy (CP) stratified by sex

\begin{tabular}{|c|c|c|c|c|}
\hline & \multirow{2}{*}{$\begin{array}{l}\text { Female } \\
\text { CP }(n=2,658)\end{array}$} & \multirow{2}{*}{$\begin{array}{l}\text { Without CP } \\
(n=2,978,467)\end{array}$} & \multirow{2}{*}{$\begin{array}{l}\text { Male } \\
\text { CP }(n=2,897)\end{array}$} & \multirow{2}{*}{$\begin{array}{l}\text { Without CP } \\
(n=2,552,899)\end{array}$} \\
\hline & & & & \\
\hline \multicolumn{5}{|l|}{ Medical conditions, $\%(95 \% \mathrm{Cl})$} \\
\hline Pain & $42.6(40.7,44.5)$ & 31.6 & $34.9(33.2,36.6)$ & 29.0 \\
\hline Fracture & $6.2(5.3,7.1)$ & 2.7 & $6.4(5.5,7.3)$ & 2.7 \\
\hline Ischemic heart disease & $4.2(3.4,5.0)$ & 2.4 & $5.8(4.9,6.7)$ & 5.1 \\
\hline Cerebrovascular disease & $7.0(6.0,8.0)$ & 1.5 & $6.7(5.8,7.6)$ & 1.7 \\
\hline Hypertensive/other cardiovascular disease & $34.4(32.6,36.2)$ & 22.2 & $40.1(38.3,41.9)$ & 29.7 \\
\hline Type 2 diabetes & $12.6(11.3,13.9)$ & 8.8 & $12.1(10.9,13.3)$ & 11.5 \\
\hline Malignant cancer & $4.3(3.5,5.1)$ & 4.1 & $3.7(3.0,4.4)$ & 3.7 \\
\hline Osteoporosis & $6.7(5.7,7.7)$ & 2.2 & $4.4(3.7,5.1)$ & 0.3 \\
\hline Osteoarthritis & $16.2(14.8,17.6)$ & 8.5 & $13.8(12.5,15.1)$ & 7.2 \\
\hline Mood affective disorders & $27.3(25.6,29.0)$ & 14.0 & $19.8(18.3,21.3)$ & 8.0 \\
\hline Anxiety disorders & $28.4(26.7,30.1)$ & 18.5 & $19.5(18.1,20.9)$ & 11.5 \\
\hline Chronic kidney disease & $3.8(3.1,4.5)$ & 1.5 & $4.8(4.0,5.6)$ & 2.2 \\
\hline Liver disease & $4.1(3.3,4.9)$ & 2.8 & $4.0(3.3,4.7)$ & 3.1 \\
\hline \multicolumn{5}{|c|}{ Health care resource utilization, mean count (SD) } \\
\hline Inpatient visits & $2.9(8.0)$ & $0.4(2.4)$ & $3.0(8.4)$ & $0.4(2.6)$ \\
\hline Outpatient and other visits & $17.9(21.3)$ & $8.4(10.7)$ & I5.5 (20.5) & $6.2(9.7)$ \\
\hline Emergency department visits & $1.0(2.0)$ & $0.3(1.0)$ & $0.9(1.9)$ & $0.3(0.8)$ \\
\hline Office visits & $7.3(6.7)$ & $4.4(4.9)$ & $6.2(6.1)$ & $3.5(4.3)$ \\
\hline Ancillary use & $9.2(27.3)$ & $1.4(5.7)$ & $9.6(26.8)$ & $1.3(5.9)$ \\
\hline \multicolumn{5}{|l|}{ Total health care costs (\$), mean (SD) } \\
\hline Standardized reimbursement & $25,844(53,939)$ & $9,421(27,485)$ & $26,084(72,855)$ & $8,179(30,822)$ \\
\hline Patient out-of-pocket & $2,097(3,016)$ & $1,325(1,945)$ & $2,011(2,798)$ & $1,122(2,010)$ \\
\hline
\end{tabular}

and obesity-related health complications.) ${ }^{34}$ Evidence suggests that children with $\mathrm{CP}$ have poor cardiorespiratory fitness ${ }^{35}$ and excess body ${ }^{4}$ and abdominal ${ }^{3}$ fat compared to children without CP. Excess body fat in childhood is a strong predictor of cardiometabolic morbidity and mortality in adulthood, ${ }^{36,37}$ and elevated abdominal fat may have a unique and profound influence on cardiometabolic disease processes. ${ }^{38}$ Further, individuals with $\mathrm{CP}$ have higher cardiovascular-related mortality compared to individuals without $\mathrm{CP}^{39,40}$ Study findings corroborate this previous literature, as we found that adults with $\mathrm{CP}$ had higher adjusted odds of all cardiometabolic disease groups in the primary analysis. However, the sensitivity analysis revealed that the increased risk for ischemic heart diseases and type 2 diabetes, but not cerebrovascular diseases, was partially accounted for by ethnicity, education level, and household annual income, which are proxy measures of socioeconomic status. Further, after stratifying by sex, women, but not men, with CP had a higher prevalence of ischemic heart diseases and type 2 diabetes compared to their respective comparison group.
Further research is needed to delineate the association of these medical conditions with socioeconomic factors and sex among adults with CP.

In the current study, we found that adults with $\mathrm{CP}$ had higher adjusted odds of pain, osteoarthritis, depressive disorders, and anxiety disorders, which is consistent with previous studies. ${ }^{5,9,10}$ Pediatric chronic pain is associated with increased risk for lifetime depressive and anxiety disorders. ${ }^{41}$ Further, osteoarthritis may exacerbate pain, which can negatively affect function and activities of daily living, ${ }^{42}$ thus posing another mechanism for adverse mental health processes and low social participation.

We also found that adults with CP had higher odds of chronic kidney and liver diseases, and to our knowledge, this is the first report of increased risk for these outcomes in the CP population. Since cardiometabolic diseases are implicated in the decline of kidney function, our findings of elevated cardiometabolic diseases may provide insight regarding mechanisms. Liver diseases are caused by a variety of factors, such as problems with immune function, 
genetic predisposition, diabetes, and excess body fat, all of which are not uncommon problems among the CP population. ${ }^{4,43}$ Interestingly, while risk for ischemic heart diseases and type 2 diabetes was partially accounted for by proxy measures of socioeconomic status, chronic kidney and liver diseases remained elevated after adjusting for these variables. Future research is needed to determine the extent to which cardiometabolic diseases are associated with chronic kidney and liver diseases among adults with CP.

Taken together, while neuromuscular and musculoskeletal problems are evident early in childhood for individuals with $\mathrm{CP}$, they lead to later-life musculoskeletal diseases $^{8,9}$ and may be responsible for initiating or exacerbating the development of several other medical conditions through direct and indirect (eg, physical activity) mechanisms. Study findings suggest that CP does not just affect one system, but has pathophysiological processes across several biological systems, which may become integrative throughout the aging process.

In the current study, we found that adults with CP had higher annual usage for all medical service categories. We also found that adults with CP incurred costs that were almost three times higher for standardized reimbursement costs and 1.7 times higher for patient out-of-pocket costs compared to adults without CP. Since high-burden medical conditions by themselves lead to greater health care costs, we statistically controlled for all medical conditions, and still found that adults with CP had excess health care costs that were 2.5 times higher for standardized reimbursement costs and 1.8 times higher for patient out-of-pocket costs. These findings suggest that factors other than high-burden medical conditions are contributing to the higher health care costs; however, there may be other costly medical conditions not examined in this study that are more prevalent among individuals with $\mathrm{CP}$.

Both unadjusted and adjusted CRs were higher for medical and pharmacy claims among adults with CP compared to adults without CP, but the CRs were more profound for medical than pharmacy for standardized reimbursement costs $(\mathrm{CR}=3.05$ and 2.42 , respectively) and patient out-of-pocket costs $(\mathrm{CR}=1.77$ and 1.40 , respectively). From a systems-level perspective, these findings suggest that health plans are accommodating some, but not all, of the medical needs of adults with CP. This is supported by the greater likelihood that adults with CP had Medicare Advantage health plans compared to adults without CP (58.5\% vs $6.9 \%)$, which may include greater cost reduction plans.
There are several limitations that need to be discussed. First, it was not possible to determine severity of CP using administrative claims data. However, the overall CP sample likely represents a healthier and higher functioning segment of the CP population. ${ }^{44}$ This may bias results to be more conservative estimates and lacks generalizability to the entire $\mathrm{CP}$ population. To be enrolled with a private health insurance plan, beneficiaries purchase their own plan or are covered through their employer, parents (up to 26 years of age), or their spouse. Individuals with pediatric-onset disabilities, such as $\mathrm{CP}$, tend to have low employment and marriage rates, ${ }^{45}$ which is likely to be more problematic with more severe forms of CP. Further research is needed to examine differences in outcomes across different databases, such as Medicare and Medicaid. Second, we used a single claim to identify CP and high-burden medical conditions. While validation studies have shown that two claims for a medical condition improves ability to identify individuals with that medical condition, ${ }^{16,46}$ single claim-based algorithms have been reported to have moderate-to-high positive predictive value $(\sim 80 \%)$ and high sensitivity $(99 \%)$ to detect pediatric-onset conditions, ${ }^{16}$ and moderate-to-high sensitivity (up to 99\%) and specificity (up to $87 \%$ ) to detect a variety of high-burden medical conditions; ${ }^{47,48}$ however, the accuracy of identifying conditions using claims data depends on the length of the study period ${ }^{49}$ and the medical condition examined. ${ }^{16,47,49,50}$ Given the short study period of 12 months to extract data and the large and robust effect sizes, the selected methodology to identify associations is likely sufficient to provide evidence of health disparities and economic burden. Third, administrative claims data are naturally subject to errors, such as inaccurate coding of medical diagnoses. Fourth, due to comorbidities associated with $\mathrm{CP}$ (eg, communication impairments, intellectual disabilities), evaluating and diagnosing certain medical conditions may be challenging for this population. Pain and mental health disorders are subject to such challenges as they require intrapersonal awareness and communication of these problems. Fifth, due to the study design, we were unable to determine the temporal sequence of high-burden medical conditions and determine which condition is manifested first, which could influence treatment strategies. Sixth, there is a possibility that the cost data are inflated compared to other health plans (eg, Medicare) since private payers could have increased services and preventive measures. 


\section{Conclusion}

Study findings from a single private payer administrative claims database suggest that young and middle-aged adults with CP have a substantially higher prevalence of several high-burden medical conditions, higher health care resource utilization, and higher health care costs compared to young and middle-aged adults without $\mathrm{CP}$. Study findings could inform health plan administrators, policymakers, and early patient- and clinical-decision making processes and strategies to maximize resourceand cost-effective treatment. For example, findings highlight the need for adopting earlier screening strategies for medical conditions, improving clinician awareness of the disease burden, and developing efficient referral processing in order to minimize the patient, caregiver, and health care burden of medical conditions. Future research is needed to determine the etiology and pathophysiology of these high-burden medical conditions and the temporal sequence of disease acquisition. For example, if musculoskeletal diseases are causing or exacerbating other high-burden medical conditions, future efforts are needed to optimize musculoskeletal health for individuals with $\mathrm{CP}$.

\section{Accessibility of protocol, raw data, and programming code}

As part of the Date Use Agreement, authors are not allowed to provide raw data. Upon reasonable request, the corresponding author will provide statistical programming code used to generate results.

\section{Acknowledgments}

This research was developed in part under a grant from the National Institute on Disability, Independent Living, and Rehabilitation Research (Mark D Peterson) (NIDILRR \#90RTHF0001-01-00). The funder had no role in the design and conduct of the study; collection, management, analysis, and interpretation of the data; preparation, review, or approval of the manuscript; and decision to submit the manuscript for publication.

\section{Disclosure}

Neil S Kamdar reports personal fees from Stanford University, Western University of the Health Sciences, and Lucent Surgical, outside the submitted work. The authors report no other conflicts of interest in this work.

\section{References}

1. Christensen D, Van Naarden Braun K, Doernberg NS, et al. Prevalence of cerebral palsy, co-occurring autism spectrum disorders, and motor functioning - Autism and developmental disabilities monitoring network, USA, 2008. Dev Med Child Neurol. 2014;56 (1):59-65. doi:10.1111/dmcn. 12268

2. Whitney DG, Singh H, Miller F, et al. Cortical bone deficit and fat infiltration of bone marrow and skeletal muscle in ambulatory children with mild spastic cerebral palsy. Bone. 2017;94:90-97. doi:10.1016/j.bone.2016.10.005

3. Whitney DG, Singh H, Zhang C, Miller F, Modlesky CM. Greater visceral fat but no difference in measures of total body fat in ambulatory children with spastic cerebral palsy compared to typically developing children. J Clin Densitom. 2018. doi:10.1016/j.jocd.2018.09.006

4. Whitney DG, Miller F, Pohlig RT, Modlesky CM. BMI does not capture the high fat mass index and low fat-free mass index in children with cerebral palsy and proposed statistical models that improve this accuracy. Int J Obes (Lond). 2018;43:82-90.

5. Whitney DG, Warschausky SA, Peterson MD. Mental health disorders and physical risk factors in children with cerebral palsy: a cross-sectional study. Dev Med Child Neurol. 2018;61:579-585.

6. Himmelmann K, Sundh V. Survival with cerebral palsy over five decades in western Sweden. Dev Med Child Neurol. 2015;57 (8):762-767. doi:10.1111/dmcn.12718

7. Day SM, Wu YW, Strauss DJ, Shavelle RM, Reynolds RJ. Change in ambulatory ability of adolescents and young adults with cerebral palsy. Dev Med Child Neurol. 2007;49(9):647-653. doi:10.1111/ j.1469-8749.2007.00647.x

8. Whitney DG, Hurvitz EA, Devlin MJ, et al. Age trajectories of musculoskeletal morbidities in adults with cerebral palsy. Bone. 2018;114:285-291. doi:10.1016/j.bone.2018.07.002

9. Whitney DG, Hurvitz EA, Ryan JM, et al. Noncommunicable disease and multimorbidity in young adults with cerebral palsy. Clin Epidemiol. 2018;10:511-519. doi:10.2147/CLEP.S159405

10. Smith KJ, Peterson MD, O'Connell NE, et al. Risk of depression and anxiety in adults with cerebral palsy. JAMA Neurol. 2019;76(3):294300. doi:10.1001/jamaneurol.2018.4147

11. Van Der Slot WM, Nieuwenhuijsen C, Van Den Berg-Emons RJ, et al. Chronic pain, fatigue, and depressive symptoms in adults with spastic bilateral cerebral palsy. Dev Med Child Neurol. 2012;54 (9):836-842. doi:10.1111/j.1469-8749.2012.04371.x

12. Peterson MD, Kamdar N, Hurvitz EA. Age-related trends in cardiometabolic disease among adults with cerebral palsy. Dev Med Child Neurol. 2019;61(4):484-489.

13. Cremer N, Hurvitz EA, Peterson MD. Multimorbidity in middle-aged adults with cerebral palsy. Am J Med. 2017;130(6):744e749744e715. doi:10.1016/j.amjmed.2016.11.044

14. Berry JG, Berry SD. Caring for patients with neurological impairment: conversations between a pediatrician and geriatrician. JAMA Pediatr. 2018;172:795. doi:10.1001/jamapediatrics.2018.1079

15. Aisen ML, Kerkovich D, Mast J, et al. Cerebral palsy: clinical care and neurological rehabilitation. Lancet Neurol. 2011;10(9):844-852. doi:10.1016/S1474-4422(11)70176-4

16. Reeves S, Garcia E, Kleyn M, et al. Identifying sickle cell disease cases using administrative claims. Acad Pediatr. 2014;14(5 Suppl): S61-S67. doi:10.1016/j.acap.2014.02.008

17. Quinones AR, Markwardt S, Botoseneanu A. Multimorbidity combinations and disability in older adults. J Gerontol A Biol Sci Med Sci. 2016;71(6):823-830. doi:10.1093/gerona/glw035

18. Salive ME. Multimorbidity in older adults. Epidemiol Rev. 2013;35:75-83. doi:10.1093/epirev/mxs009

19. Whiteford HA, Degenhardt L, Rehm J, et al. Global burden of disease attributable to mental and substance use disorders: findings from the Global burden of disease study 2010. Lancet. 2013;382 (9904):1575-1586. doi:10.1016/S0140-6736(13)61611-6 
20. Murray CJ, Vos T, Lozano R, et al. Disability-adjusted life years (DALYs) for 291 diseases and injuries in 21 regions, 1990-2010: a systematic analysis for the Global burden of disease study 2010 . Lancet. 2012;380(9859):2197-2223. doi:10.1016/S0140-6736(12) 61689-4

21. GBD 2016 Causes of Death Collaborators. Global, regional, and national age-sex specific mortality for 264 causes of death, 1980-2016: a systematic analysis for the Global burden of disease study 2016. Lancet. 2017;390(10100):1151-1210. doi:10.1016/ S0140-6736(17)32152-9

22. GBD 2017 DALYs and HALE Collaborators. Global, regional, and national disability-adjusted life-years (DALYs) for 359 diseases and injuries and healthy life expectancy (HALE) for 195 countries and territories, 1990-2017: a systematic analysis for the Global burden of disease study 2017. Lancet. 2018;392(10159):1859-1922. doi:10.1016/S0140-6736(18)32335-3

23. GBD 2017 Disease and Injury Incidence and Prevalence Collaborators. Global, regional, and national incidence, prevalence, and years lived with disabilitity for 354 diseases and injuries for 195 countries and territories, 1990-2017: a systematic analysis for the Global burden of disease study 2017. Lancet. 2018;392 (10159):1789-1858.

24. Burge R, Dawson-Hughes B, Solomon DH, Wong JB, King A, Tosteson A. Incidence and economic burden of osteoporosis-related fractures in the United States, 2005-2025. J Bone Miner Res. 2007;22(3):465-475. doi:10.1359/jbmr.061113

25. Zhang J, Yu KF. What's the relative risk? A method of correcting the odds ratio in cohort studies of common outcomes. Jama. 1998;280 (19):1690-1691.

26. Barber J, Thompson S. Multiple regression of cost data: use of generalised linear models. J Health Serv Res Policy. 2004;9 (4):197-204. doi:10.1258/1355819042250249

27. Diehr P, Yanez D, Ash A, Hornbrook M, Lin DY. Methods for analyzing health care utilization and costs. Annu Rev Public Health. 1999;20:125-144. doi:10.1146/annurev.publhealth.20.1.125

28. Mantopoulos T, Mitchell PM, Welton NJ, McManus R, Andronis L. Choice of statistical model for cost-effectiveness analysis and covariate adjustment: empirical application of prominent models and assessment of their results. Eur $J$ Health Econ. 2016;17 (8):927-938. doi:10.1007/s10198-015-0731-8

29. Kruse M, Michelsen SI, Flachs EM, Bronnum-Hansen H, Madsen M, Uldall P. Lifetime costs of cerebral palsy. Dev Med Child Neurol. 2009;51(8):622-628. doi:10.1111/j.1469-8749.2008.03190.x

30. Moreau NG, Falvo MJ, Damiano DL. Rapid force generation is impaired in cerebral palsy and is related to decreased muscle size and functional mobility. Gait Posture. 2012;35(1):154-158. doi:10.1016/j.gaitpost.2011.08.027

31. Stackhouse SK, Binder-Macleod SA, Lee SC. Voluntary muscle activation, contractile properties, and fatigability in children with and without cerebral palsy. Muscle Nerve. 2005;31(5):594-601. doi:10.1002/mus.20302

32. Modlesky CM, Whitney DG, Singh H, Barbe MF, Kirby JT, Miller F. Underdevelopment of trabecular bone microarchitecture in the distal femur of nonambulatory children with cerebral palsy becomes more pronounced with distance from the growth plate. Osteoporos Int. 2015;26(2):505-512. doi:10.1007/s00198-014-2873-4

33. Herskind A, Ritterband-Rosenbaum A, Willerslev-Olsen M, et al. Muscle growth is reduced in 15-month-old children with cerebral palsy. Dev Med Child Neurol. 2016;58(5):485-491. doi:10.1111/ dmcn. 12950

34. Whitney DG, Peterson MD, Devlin MJ, Caird MS, Hurvitz EA, Modlesky CM. Bone marrow fat physiology in relation to skeletal metabolism and cardiometabolic disease risk in children with cerebral palsy. Am J Phys Med Rehabil. 2018;97:911-919. doi:10.1097/ PHM.0000000000000981
35. Garcia CC, Alcocer-Gamboa A, Ruiz MP, et al. Metabolic, cardiorespiratory, and neuromuscular fitness performance in children with cerebral palsy: a comparison with healthy youth. J Exerc Rehabil. 2016;12(2):124-131. doi:10.12965/jer.1632552.276

36. Franks PW, Hanson RL, Knowler WC, Sievers ML, Bennett PH, Looker HC. Childhood obesity, other cardiovascular risk factors, and premature death. $N$ Engl J Med. 2010;362(6):485-493. doi:10.1056/NEJMoa0904130

37. Bibbins-Domingo K, Coxson P, Pletcher MJ, Lightwood J, Goldman L. Adolescent overweight and future adult coronary heart disease. $N$ Engl J Med. 2007;357(23):2371-2379. doi:10.1056/ NEJMsa073166

38. Philipsen A, Jorgensen ME, Vistisen D, et al. Associations between ultrasound measures of abdominal fat distribution and indices of glucose metabolism in a population at high risk of type 2 diabetes: the ADDITION-PRO study. PLoS One. 2015;10(4):e123062. doi:10.1371/journal.pone.0123062

39. Strauss D, Cable W, Shavelle R. Causes of excess mortality in cerebral palsy. Dev Med Child Neurol. 1999;41(9):580-585.

40. Ryan JM, Peterson MD, Ryan N, et al. Mortality due to cardiovascular disease, respiratory disease, and cancer in adults with cerebral palsy. Dev Med Child Neurol. 2019. doi:10.1111/dmcn.14176

41. Noel M, Groenewald CB, Beals-Erickson SE, Gebert JT, Palermo TM. Chronic pain in adolescence and internalizing mental health disorders: a nationally representative study. Pain. 2016;157 (6):1333-1338. doi:10.1097/j.pain.0000000000000522

42. Murphy SL, Schepens Niemiec S, Lyden AK, Kratz AL. Pain, fatigue, and physical activity in osteoarthritis: the moderating effects of pain- and fatigue-related activity interference. Arch Phys Med Rehabil. 2016;97(9 Suppl):S201-S209. doi:10.1016/j.apmr.2015.05.025

43. Lisovska N, Daribayev Z, Lisovskyy Y, Kussainova K, Austin L, Bulekbayeva S. Pathogenesis of cerebral palsy through the prism of immune regulation of nervous tissue homeostasis: literature review. Childs Nerv Syst. 2016;32(11):2111-2117. doi:10.1007/s00381-0163245-5

44. Whitney DG, Alford AI, Devlin MJ, Caird MS, Hurvitz EA, Peterson MD. Adults with cerebral palsy have higher prevalence of fracture compared with adults without cerebral palsy independent of osteoporosis and cardiometabolic diseases. J Bone Miner Res. 2019. doi:10.1002/jbmr.3694

45. Tumin D. Marriage trends among Americans with childhood-onset disabilities, 1997-2013. Disabil Health J. 2016;9(4):713-718. doi:10.1016/j.dhjo.2016.05.004

46. Kerr EA, McGlynn EA, Van Vorst KA, Wickstrom SL. Measuring antidepressant prescribing practice in a health care system using administrative data: implications for quality measurement and improvement. Jt Comm J Qual Improv. 2000;26(4):203-216.

47. Doktorchik C, Patten S, Eastwood C, et al. Validation of a case definition for depression in administrative data against primary chart data as a reference standard. BMC Psychiatry. 2019;19(1):9. doi:10.1186/s12888-019-2104-9

48. Kurdyak P, Lin E, Green D, Vigod S. Validation of a population-based algorithm to detect chronic psychotic illness. Can J Psychiatry. 2015;60 (8):362-368. doi:10.1177/070674371506000805

49. Leslie WD, Lix LM, Yogendran MS. Validation of a case definition for osteoporosis disease surveillance. Osteoporos Int. 2011;22 (1):37-46. doi:10.1007/s00198-010-1225-2

50. Noyes K, Liu H, Lyness JM, Friedman B. Medicare beneficiaries with depression: comparing diagnoses in claims data with the results of screening. Psychiatr Serv. 2011;62(10):1159-1166. doi:10.1176/ ps.62.10.pss6210_1159 


\section{Publish your work in this journal}

Clinical Epidemiology is an international, peer-reviewed, open access, online journal focusing on disease and drug epidemiology, identification of risk factors and screening procedures to develop optimal preventative initiatives and programs. Specific topics include: diagnosis, prognosis, treatment, screening, prevention, risk factor modification,

Submit your manuscript here: https://www.dovepress.com/clinical-epidemiology-journal systematic reviews, risk \& safety of medical interventions, epidemiology \& biostatistical methods, and evaluation of guidelines, translational medicine, health policies \& economic evaluations. The manuscript management system is completely online and includes a very quick and fair peer-review system, which is all easy to use. 